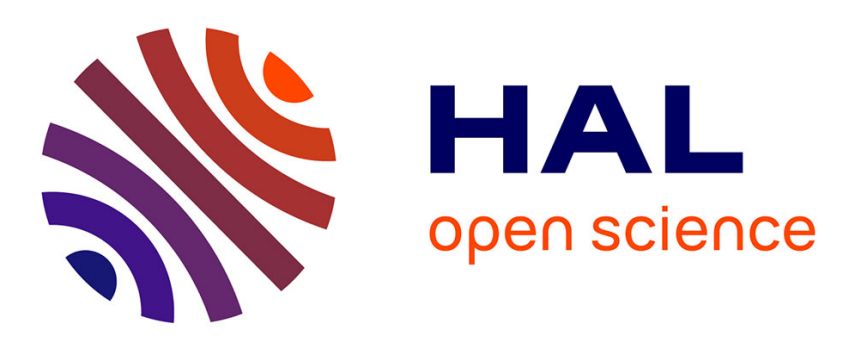

\title{
Déterminants urinaires de la lithogénèse urique : rôle de la graisse périrénale chez les sujets en surpoids ou obèses
}

Florence François Chalmin

\section{To cite this version:}

Florence François Chalmin. Déterminants urinaires de la lithogénèse urique : rôle de la graisse périrénale chez les sujets en surpoids ou obèses. Sciences du Vivant [q-bio]. 2017. dumas-01681164

\section{HAL Id: dumas-01681164 https://dumas.ccsd.cnrs.fr/dumas-01681164}

Submitted on 11 Jan 2018

HAL is a multi-disciplinary open access archive for the deposit and dissemination of scientific research documents, whether they are published or not. The documents may come from teaching and research institutions in France or abroad, or from public or private research centers.
L'archive ouverte pluridisciplinaire HAL, est destinée au dépôt et à la diffusion de documents scientifiques de niveau recherche, publiés ou non, émanant des établissements d'enseignement et de recherche français ou étrangers, des laboratoires publics ou privés. 
Université de Nice Sophia - Antipolis

Faculté de Médecine de Nice

Année 2016 - 2017

Déterminants urinaires de la lithogénèse urique :

Rôle de la graisse périrénale chez les sujets en surpoids ou obèses.

\section{Thèse de Médecine}

Pour l'obtention du grade de Docteur en Médecine (Diplôme d'état)

Présentée et soutenue publiquement le mardi 21 mars 2017 par

\section{Mme Florence FRANCOIS \\ épouse CHALMIN}

Née le 1er Décembre 1988 à Wagga Wagga (Australie)

Interne des Hôpitaux de Nice

Spécialité Néphrologie

Composition du jury:

Mr le Professeur Vincent ESNAULT

Président

Mr le Professeur Bertrand CANIVET

Assesseur

Mr le Docteur Mohamed Shariful ISLAM

Assesseur

Mr le Docteur Charles-Paul RAFFAELLI

Assesseur

Mr le Maître de Conférences Guillaume FAVRE

Directeur 


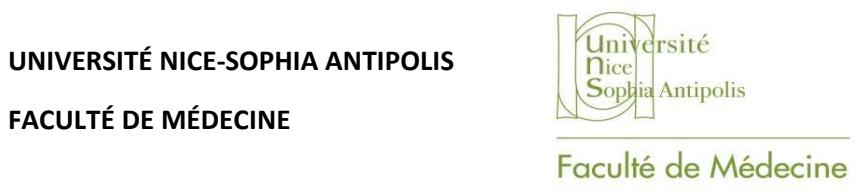

\section{Liste des professeurs au 1er septembre 2016 à la Faculté de Médecine de Nice}

\section{Doyen}

Vice-Doyen

Assesseurs

Conservateur de la bibliothèque

Directrice administrative des services

Doyens Honoraires

\section{BAQUÉ Patrick}

M. BOILEAU Pascal

M. ESNAULT Vincent

M. CARLES Michel

Mme BREUIL Véronique

M. MARTY Pierre

Mme DE LEMOS Annelyse

Mme CALLEA Isabelle

M. AYRAUD Noël

M. RAMPAL Patrick

M. BENCHIMOL Daniel

\section{Professeurs Honoraires}

M ALBERTINI Marc

M. BALAS Daniel

M. BATT Michel

M. BLAIVE Bruno

M. BOQUET Patrice

M. BOURGEON André

M. BOUTTÉ Patrick

M. BRUNETON Jean-Noël

Mme BUSSIERE Françoise

M. CAMOUS Jean-Pierre

M. CANIVET Bertrand

M. CASSUTO Jill-patrice

M. CHATEL Marcel

M. COUSSEMENT Alain

Mme CRENESSE Dominique

M. DARCOURT GuY

M. DELLAMONICA Pierre

M. DELMONT Jean

M. DEMARD François

M. DOLISI Claude

$M$. FRANCO Alain

M. FREYCHET Pierre

M. GÉRARD Jean-Pierre

M. GILLET Jean-Yves

M. GRELLIER Patrick

M. GRIMAUD Dominique
M. HARTER Michel

M. INGLESAKIS Jean-André

M. JOURDAN Jacques

M. LALANNE Claude-Michel

M. LAMBERT Jean-Claude

M. LAZDUNSKI Michel

M. LEFEBVRE Jean-Claude

M. LE BAS Pierre

M. LE FICHOUX Yves

Mme LEBRETON Elisabeth

M. LOUBIERE Robert

M. MARIANI Roger

M. MASSEYEFF René

M. MATTEI Mathieu

M. MOUIEL Jean

Mme MYQUEL Martine

M. OLLIER Amédée

M. ORTONNE Jean-Paul

M. SAUTRON Jean Baptiste

M. SCHNEIDER Maurice

$M$. TOUBOL Jacques

M. TRAN Dinh Khiem

$M$ VAN OBBERGHEN Emmanuel

M. ZIEGLER Gérard 


UNIVERSITÉ NICE-SOPHIA ANTIPOLIS
FACULTÉ DE MÉDECINE

Liste des professeurs au 1er septembre 2016 à la Faculté de Médecine de Nice

Mlle ALLINE Madeleine

M.C.U. Honoraires

M. ARNOLD Jacques
M. BASTERIS Bernard
MIle CHICHMANIAN Rose-Marie
Mme DONZEAU Michèle
M. EMILIOZZI Roméo
M. FRANKEN Philippe
M. GASTAUD Marcel
M.GIRARD-PIPAU Fernand
M. GIUDICELLI Jean
M. MAGNÉ Jacques
Mme MEMRAN Nadine
M. MENGUAL Raymond
M. PHILIP Patrick
M. POIRÉE Jean-Claude
Mme ROURE Marie-Claire




\section{UNIVERSITÉ NICE-SOPHIA ANTIPOLIS FACULTÉ DE MÉDECINE}

Liste des professeurs au 1er septembre 2016 à la Faculté de Médecine de Nice

\section{PROFESSEURS CLASSE EXCEPTIONNELLE}

\begin{tabular}{|c|c|}
\hline M. & AMIEL Jean \\
\hline M. & BENCHIMOL Daniel \\
\hline И. & BOILEAU Pascal \\
\hline M. & DARCOURT Jacques \\
\hline M. & DESNUELLE Claude \\
\hline Mme & EULLER-ZIEGLER Liana \\
\hline M. & FENICHEL Patrick \\
\hline M. & FUZIBET Jean-Gabriel \\
\hline M. & GASTAUD Pierre \\
\hline M. & GILSON Éric \\
\hline M. & HASSEN KHODJA Reda \\
\hline M. & HÉBUTERNE Xavier \\
\hline M. & HOFMAN Paul \\
\hline M. & LACOUR Jean-Philippe \\
\hline M. & LEFTHERIOTIS Geogres \\
\hline M. & MARTY Pierre \\
\hline M. & MICHIELS Jean-François \\
\hline M. & MOUROUX Jérôme \\
\hline Mme & PAQUIS Véronique \\
\hline M. & PAQUIS Philippe \\
\hline M. & PRINGUEY Dominique \\
\hline M. & QUATREHOMME Gérald \\
\hline M. & RAUCOULES-AIMÉ MarC \\
\hline M. & ROBERT Philippe \\
\hline M. & SANTINI Joseph \\
\hline M. & THYSS Antoine \\
\hline M. & TRAN Albert \\
\hline
\end{tabular}

\author{
Urologie (52.04) \\ Chirurgie Générale (53.02) \\ Chirurgie Orthopédique et Traumatologique (50.02) \\ Biophysique et Médecine Nucléaire (43.01) \\ Biologie Cellulaire (44.03) \\ Rhumatologie (50.01) \\ Biologie du Développement et de la Reproduction (54.05) \\ Médecine Interne (53.01) \\ Ophtalmologie (55.02) \\ Biologie Cellulaire (44.03) \\ Chirurgie Vasculaire (51.04) \\ Nutrition (44.04) \\ Anatomie et Cytologie Pathologiques (42.03) \\ Dermato-Vénéréologie (50.03) \\ Physiologie- médecine vasculaire \\ Parasitologie et Mycologie (45.02) \\ Anatomie et Cytologie Pathologiques (42.03) \\ Chirurgie Thoracique et Cardiovasculaire (51.03) \\ Génétique (47.04) \\ Neurochirurgie (49.02) \\ Psychiatrie d'Adultes (49.03) \\ Médecine Légale et Droit de la Santé (46.03) \\ Anesthésie et Réanimation Chirurgicale (48.01) \\ Psychiatrie d'Adultes (49.03) \\ O.R.L. (55.01) \\ Cancérologie, Radiothérapie (47.02) \\ Hépato Gastro-entérologie (52.01)
}




\section{UNIVERSITÉ NICE-SOPHIA ANTIPOLIS FACULTÉ DE MÉDECINE}

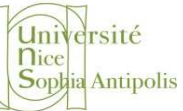

Faculté de Médecine

Liste des professeurs au 1er septembre 2016 à la Faculté de Médecine de Nice

\section{PROFESSEURS PREMIERE CLASSE}

$\begin{array}{ll}\text { Mme } & \text { ASKENAZY-GITTARD Florence } \\ \text { M. } & \text { BAQUÉ Patrick } \\ \text { M. } & \text { BARRANGER Emmanuel } \\ \text { M. } & \text { BÉRARD Étienne } \\ \text { M. } & \text { BERNARDIN Gilles } \\ \text { Mme } & \text { BLANC-PEDEUTOUR Florence } \\ \text { M. } & \text { BONGAIN André } \\ \text { M. } & \text { CASTILLO Laurent } \\ \text { M. } & \text { DE PERETTI Fernand } \\ \text { M. } & \text { DRICI Milou-Daniel } \\ \text { M. } & \text { ESNAULT Vincent } \\ \text { M. } & \text { FERRARI Émile } \\ \text { M. } & \text { FERRERO Jean-Marc } \\ \text { M. } & \text { GIBELIN Pierre } \\ \text { M. } & \text { GUGENHEIM Jean } \\ \text { M. } & \text { HANNOUN-LEVI Jean-Michel } \\ \text { Mme } & \text { ICHAI Carole } \\ \text { M. } & \text { LONJON Michel } \\ \text { M. } & \text { MARQUETTE Charles-Hugo } \\ \text { M. } & \text { MOUNIER Nicolas } \\ \text { M. } & \text { PADOVANI Bernard } \\ \text { M. } & \text { PRADIER Christian } \\ \text { Mme } & \text { RAYNAUD Dominique } \\ \text { M. } & \text { ROSENTHAL Éric } \\ \text { M. } & \text { SCHNEIDER Stéphane } \\ \text { M. } & \text { STACCINI Pascal } \\ \text { M. } & \text { THOMAS Pierre } \\ & \end{array}$

Pédopsychiatrie (49.04)

Anatomie - Chirurgie Générale (42.01)

Gynécologie Obstétrique (54.03)

Pédiatrie (54.01)

Réanimation Médicale (48.02)

Cancérologie - Génétique (47.02)

Gynécologie-Obstétrique (54.03)

O.R.L. (55.01)

Anatomie-Chirurgie Orthopédique (42.01)

Pharmacologie Clinique (48.03)

Néphrologie (52-03)

Cardiologie (51.02)

Cancérologie ; Radiothérapie (47.02)

Cardiologie (51.02)

Chirurgie Digestive (52.02)

Cancérologie ; Radiothérapie (47.02)

Anesthésiologie et Réanimation Chirurgicale (48.01)

Neurochirurgie (49.02)

Pneumologie (51.01)

Cancérologie, Radiothérapie (47.02)

Radiologie et Imagerie Médicale (43.02)

Épidémiologie, Économie de la Santé et Prévention (46.01)

Hématologie (47.01)

Médecine Interne (53.01)

Nutrition (44.04)

Biostatistiques et Informatique Médicale (46.04)

Neurologie (49.01) 


\section{UNIVERSITÉ NICE-SOPHIA ANTIPOLIS FACULTÉ DE MÉDECINE}

Liste des professeurs au 1er septembre 2016 à la Faculté de Médecine de Nice

\section{PROFESSEURS DEUXIEME CLASSE}

\begin{tabular}{ll} 
M. & BAHADORAN Philippe \\
Mme & BAILLIF Stéphanie \\
M. & BENIZRI Emmanuel \\
M. & BENOIT Michel \\
M. & BREAUD Jean \\
Mlle & BREUIL Véronique \\
M. & CARLES Michel \\
M. & CHEVALIER Nicolas \\
M. & CHEVALLIER Patrick \\
Mme & CHINETTI Giulia \\
M. & DELLAMONICA Jean \\
M. & DELOTTE Jérôme \\
M. & FONTAINE Denys \\
M. & FOURNIER Jean-Paul \\
MIle & GIORDANENGO Valérie \\
M. & GUÉRIN Olivier \\
M. & IANNELLI Antonio \\
M & JEAN BAPTISTE Elixène \\
M. & LEVRAUT Jacques \\
M. & PASSERON Thierry \\
M. & PICHE Thierry \\
M. & ROGER Pierre-Marie \\
M. & ROHRLICH Pierre \\
M. & ROUX Christian \\
M. & RUIMY Raymond \\
Mme & SACCONI Sabrina \\
M. & SADOUL Jean-Louis \\
M. & TROJANI Christophe \\
M. & VENISSAC Nicolas \\
& \\
\hline
\end{tabular}

Cytologie et Histologie (42.02)

Ophtalmologie (55.02)

Chirurgie Générale (53.02)

Psychiatrie (49.03)

Chirurgie Infantile (54-02)

Rhumatologie (50.01)

Anesthésiologie Réanimation (48.01)

Endocrinologie, Diabète et Maladies Métaboliques (54.04)

Radiologie et Imagerie Médicale (43.02)

Biochimie-Biologie Moléculaire (44.01)

réanimation médicale (48.02)

Gynécologie-obstétrique (54.03)

Neurochirurgie (49.02)

Thérapeutique (48-04)

Bactériologie-Virologie (45.01)

Gériatrie (48.04)

Chirurgie Digestive (52.02)

Chirurgie vasculaire (51.04)

Anesthésiologie et Réanimation Chirurgicale (48.01)

Dermato-Vénéréologie (50-03)

Gastro-entérologie (52.01)

Maladies Infectieuses; Maladies Tropicales (45.03)

Pédiatrie (54.01)

rhumatologie (50.01)

Bactériologie-virologie (45.01)

Neurologie (49.01)

Endocrinologie, Diabète et Maladies Métaboliques (54.04)

Chirurgie Orthopédique et Traumatologique (50.02)

Chirurgie Thoracique et Cardiovasculaire (51.03) 


\section{UNIVERSITÉ NICE-SOPHIA ANTIPOLIS FACULTÉ DE MÉDECINE \\ Université \\ Sophia Antipolis \\ Faculté de Médecine}

Liste des professeurs au 1er septembre 2016 à la Faculté de Médecine de Nice

\section{PROFESSEUR DES UNIVERSITÉS}

M. HOFLIGER Philippe Médecine Générale

PROFESSEURS AGRÉGÉS

$\begin{array}{lll}\text { Mme } & \text { LANDI Rebecca } & \text { Anglais } \\ \text { Mme } & \text { ROSE Patricia } & \text { Anglais }\end{array}$

MAITRES DE CONFÉRENCES DES UNIVERSITÉS - PRATICIENS HOSPITALIERS

Mme ALUNNI Véronique

M. AMBROSETTI Damien

Mme BANNWARTH Sylvie

$M$. BENOLIEL José

Mme BERNARD-POMIER Ghislaine

Mme BUREL-VANDENBOS Fanny

M. DARMON David

M. DOGLIO Alain

M DOYEN Jérôme

M FAVRE Guillaume

M. FOSSE Thierry

M. GARRAFFO Rodolphe

Mme GIOVANNINI-CHAMI Lisa

Mme HINAULT Charlotte

M. HUMBERT Olivier

Mme LAMY Brigitte

Mme LEGROS Laurence

Mme LONG-MIRA Elodie

Mme MAGNIÉ Marie-Noëlle

Mme MOCERI Pamela

Mme MUSSO-LASSALLE Sandra

M. NAÏMI Mourad

Mme POMARES Christelle

Mme SEITZ-POLSKI barbara

M. TESTA Jean

M. TOULON Pierre
Médecine Légale et Droit de la Santé (46.03)

Cytologie et Histologie (42.02)

Génétique (47.04)

Biophysique et Médecine Nucléaire (43.01)

Immunologie (47.03)

Anatomie et Cytologie pathologiques (42.03)

Médecine Générale

Bactériologie-Virologie (45.01)

Radiothérapie (47.02)

Néphrologie (52.03)

Bactériologie-Virologie-Hygiène (45.01)

Pharmacologie Fondamentale (48.03)

Pédiatrie (54.01)

Biochimie et biologie moléculaire (44.01)

Biophysique et Médecine Nucléaire (43.01)

Bactérilogie-virologie ( 45.01)

Hématologie et Transfusion (47.01)

Cytologie et Histologie (42.02)

Physiologie (44.02)

Cardiologie (51.02)

Anatomie et Cytologie pathologiques (42.03)

Biochimie et Biologie moléculaire (44.01)

Parasitologie et mycologie (45.02)

Immunologie (47.03)

Épidémiologie Économie de la Santé et Prévention (46.01)

Hématologie et Transfusion (47.01) 


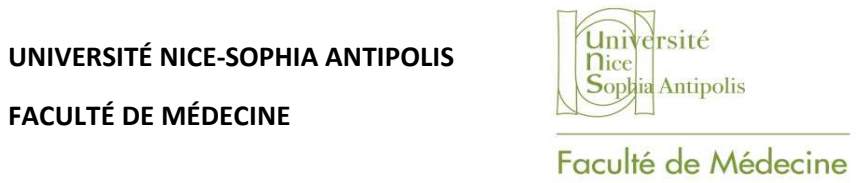

Liste des professeurs au 1er septembre 2016 à la Faculté de Médecine de Nice

\section{PRATICIEN HOSPITALIER UNIVERSITAIRE}

M. DURAND Matthieu Urologie (52.04)

PROFESSEURS ASSOCIÉS

$\begin{array}{lll}\text { M. GARDON Gilles } & \text { Médecine Générale } \\ \text { M. } & \text { GONZALEZ Jean-François } & \begin{array}{l}\text { Chirurgie Orthopédique } \\ \text { Médecine Générale }\end{array} \\ \text { M. } & \text { PAPA Michel } & \begin{array}{l}\text { Anatomie-Cytologie } \\ \text { MELLS Michael }\end{array} \\ \text { MAITRES DE CONFÉRENCES ASSOCIÉS } & \\ \text { M } & \text { BALDIN Jean-Luc } & \text { Médecine Générale } \\ \text { Mme } & \text { CASTA Céline } & \text { Médecine Générale } \\ \text { Mme } & \text { MONNIER Brigitte } & \text { Médecine Générale }\end{array}$

PROFESSEURS CONVENTIONNÉS DE L'UNIVERSITÉ

$\begin{array}{lll}\text { M. } & \text { BERTRAND François } & \text { Médecine Interne } \\ \text { M. } & \text { BROCKER Patrice } & \text { Médecine Interne Option Gériatrie } \\ \text { M. } & \text { CHEVALLIER Daniel } & \text { Urologie } \\ \text { Mme } & \text { FOURNIER-MEHOUAS Manuella } & \text { Médecine Physique et Réadaptation } \\ \text { M. } & \text { JAMBOU Patrick } & \text { Coordination prélèvements d'organes } \\ \text { M. } & \text { ODIN Guillaume } & \text { Chirurgie maxilo-faciale } \\ \text { M. } & \text { PEYRADE Frédéric } & \text { Onco-Hématologie } \\ \text { M. } & \text { PICCARD Bertrand } & \text { Psychiatrie } \\ \text { M. } & \text { QUARANTA Jean-François } & \text { Santé Publique }\end{array}$




\section{TABLE DES MATIÈRES}

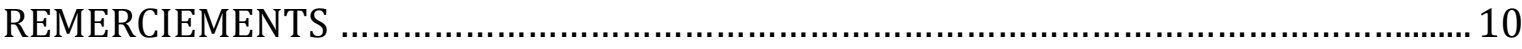

RÉSUMÉ





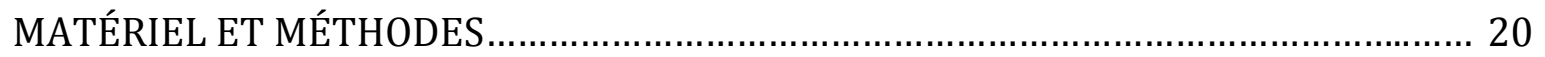

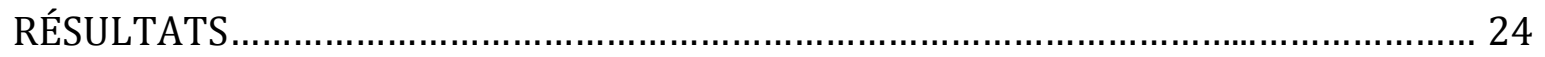

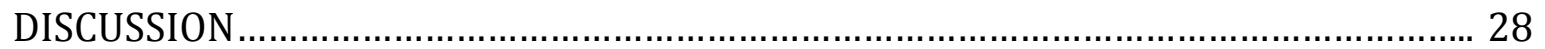

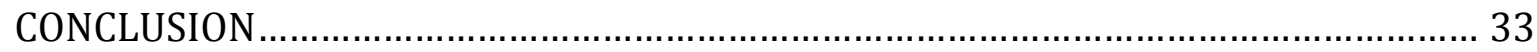



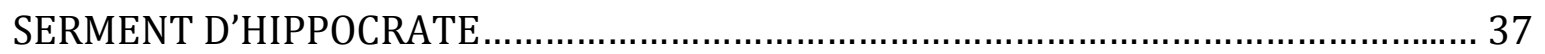




\section{REMERCIEMENTS AUX MEMBRES DU JURY}

\section{À mon maître et président du jury Monsieur le Professeur Vincent ESNAULT}

Merci de me faire l'honneur de présider mon jury de thèse. Votre enseignement au cours de mon externat et de mon internat, mais aussi votre implication auprès des patients a su éveiller mon intérêt pour la Néphrologie. Je vous en remercie car je m'épanouis aujourd'hui dans cette voie.

\section{À mon maître et juge Monsieur le Professeur Bertrand CANIVET}

Même si nos chemins se croisent plus souvent en dehors de la faculté, vous avez été mon juge à mon examen de fin de deuxième cycle et ce soir vous jugez mon travail de thèse. Je vous en remercie.

\section{À mon maître et juge Monsieur le Docteur Mohamed Shariful ISLAM}

Merci d'avoir accepté de juger mon travail. Tu as su me donner le goût de la dialyse et m'a toujours encouragée à perfectionner ma connaissance dans le domaine. Tu m'as accordé ta confiance depuis le début et je ferai tout pour continuer à la mériter.

\section{À mon maître et juge Monsieur le Docteur Charles-Paul RAFFAELLI}

Je vous remercie d'avoir accepté de faire partie de mon jury. Votre expertise et votre disponibilité font de vous une personne très respectée dans le service de Néphrologie.

\section{À mon maître et directeur de thèse Monsieur le Docteur Guillaume FAVRE}

Merci de m'avoir proposé ce projet et de m'avoir guidée dans son écriture. Au delà de mon apprentissage de la prise en charge des lithiases, tu m'as initiée à l'élaboration d'un projet scientifique et je t'en remercie. Je te remercie aussi pour ton compagnonnage dans le monde de la dialyse péritonéale et pour la confiance que tu m'accordes auprès de tes patients. 


\section{REMERCIEMENTS AUX ÉQUIPES MÉDICALES}

\section{Aux équipes des Soins Intensifs de Néphrologie}

De mes premiers pas de faisant fonction d'interne il y a 5 ans à aujourd'hui, vous m'avez bien vu grandir. Je pense qu'en dehors de ma famille, vous êtes les seuls à m'avoir vu perdre patience (dans une situation adaptée bien sur ;). Mais ces cinq ans ont surtout été remplis de nombreuses joies (dont quelques mariages) et de multiples " cups of tea »!! Je suis heureuse de savoir que je continuerai à travailler avec vous car vous êtes vraiment une équipe en or, dynamique, sérieuse et ne ménageant pas ses efforts quand je vous surcharge (un peu) de travail.

\section{Aux équipes du Centre d'Hémodialyse}

Merci pour tout ce que vous m'avez appris (et je parle aussi des quelques jeunes retraitées qui nous ont quittés récemment et qui sauront se reconnaître). Vous avez su me surprendre par votre dévouement pendant ces quelques mois difficiles l'année dernière. Je suis ravie de savoir que je travaillerai avec vous tous les jours très bientôt.

\section{Aux équipes de Néphrologie Clinique}

Nous avons partagé beaucoup de moments ensemble. Bientôt vos nuits et week-ends ne seront plus hantés par ma petite voix qui dit «au fait, j'ai accepté (encore) une petite entrée » (mais dit avec le sourire bien sûr que vous n'avez jamais manqué de me rendre !)

\section{Aux équipes de Transplantation Rénale}

Mon semestre avec vous a été très agréable. Je n'oublierais jamais le SAV de la Transplantation.

\section{Aux équipes de l’Hôpital de Jour et de Dialyse Péritonéale}

Merci pour votre compréhension et adaptabilité ce dernier semestre. J'ai découvert en vous des filles motivées et agréables avec qui je compte bien continuer à collaborer.

À Alex et Thomas, je suis ravie de pouvoir continuer à travailler avec vous car je sais que nous partageons le même calme avec pour objectifs communs le bien-être de nos patients et notre plaisir au travail et dans la vie.

À Marine et Aimele, j'admire votre enthousiasme et énergie dans le service et apprécie énormément vos personnalités lors de nos soirées hors de l'hôpital.

À Ahmed et Emmanuelle, merci pour tout ce que vous m'avez appris au cours de mon semestre en transplantation. J'espère que vous vous épanouissez tous les deux dans vos nouvelles vies. 
À Madame Cassuto et Mme Albano, merci pour votre enseignement en transplantation, votre disponibilité et pédagogie même en pleine nuit.

Au Professeur Jourdan, toujours là pour ses patients.

À Céline Fernandez, qui savait où et comment trouver toutes les informations en quelques minutes, sans qui ce travail aurait été bien plus difficile.

À nos diététiciennes, Carole et Delphine, sur qui une partie de ce travail repose et avec qui j'adore travailler.

Aux services d'Urologie du CHU de Nice et du CHPG de Monaco, ainsi qu'aux laboratoires de biochimie du CHU de Nice et d'analyse spectrophotométrique infra-rouge de Monaco, qui nous ont permis de recruter tous les patients de ce travail et d'analyser leurs données.

Aux équipes des Services de Réanimation Polyvalente d'Antibes et de Cardiologie de Cannes qui m’ont énormément appris (sur la médecine, mais aussi sur les marathons, les ultra-trails et sur les apéros entre les îles de Lérins). Votre bonne humeur au travail sera pour moi un souvenir irremplaçable.

Aux équipes de Médecine Interne de l'Archet, qui m'ont accueillie alors que j'étais encore une mini-interne et qui m'ont appris une grande partie des gestes techniques que je fais aujourd'hui avec sérénité.

À Thierry, mon super co-interne qui n'en manque pas une pour nous faire rire et qui essaye de se prendre pour mon chef depuis 4 mois.

À Soso, qui est une super amie depuis 10 ans.

Aux Néphro-Girls, Aurélie, Audrey, Hajar et Emilie (et probablement bientôt Marion et Claire)

Et aux Néphro-Boys, Joël, Gabriel, Tristan et Thibault (vous n'avez jamais entendu vos oreilles siffler pendant nos soirées filles ?)

A tous les co-internes avec qui j'ai partagé ce bout de chemin. On a beaucoup ri, on a un peu pleuré et on a surtout beaucoup appris: Grég, Sarah, Maria, Yann, Pierre, Juliette.

A nos patients, puisqu'après tout, si on est là ce soir, c'est d'abord pour eux. 


\section{REMERCIEMENTS À LA FAMILLE ET AUX AMIS}

À mon adorable mari Jérémy qui me soutient et me conseille au quotidien. Tout ce parcours médical, je l'ai fait à tes côtés, avec ton aide. Je suis très fière de toi, pour tes qualités de mari, de père de famille et pour le super pneumologue que tu es en train de devenir.

À notre fils Clément arrivé dans nos vies il y a un peu plus d'un an et qui les a bouleversées à tous jamais. Après avoir assisté à la thèse de ton papa, ce soir tu arpenteras une nouvelle fois les bancs de la faculté de médecine pour la thèse de ta maman.

À mes parents et beaux-parents, Patrick, Catherine, Daniel et Chantal. Merci pour votre soutien pendant ce long cursus, même quand j'avais des projets un peu fous. Merci aussi pour toute votre aide avec Clément ces derniers mois pour me libérer un peu de temps libre pour préparer ce travail.

À ma sœur Chloé qui ne râle jamais quand on lui envoie un dossier de 30 pages (du mémoire de comptabilité gestion à la thèse de médecine) à corriger avant demain.

A mon (doublement) beau-frère Baptiste avec qui j'ai souvent passé des après-midis de révision. Ne t'inquiète pas, je trouverai bien un DU ou deux pour t'accompagner pendant tes révisions d'ostéopathie.

A mon frère Thomas et à sa belle Laetitia, j'espère que vous vous amusez vous bien chez les kiwis car vous nous manquez beaucoup!

A Clémence qui est une très bonne amie depuis des années et qui a récemment pris un rôle plus important dans notre famille.

A Pierre Marc, Laura, Ghislain, Sonia (tu es vraiment partout, doublement merci !) et Fred. 
Déterminants urinaires de la lithogénèse urique :

Rôle de la graisse périrénale chez les sujets en surpoids ou obèses. 


\section{RÉSUMÉ :}

INTRODUCTION : Les lithiases urinaires affectent 5 à $10 \%$ de la population dans les pays industrialisés. Notre hypothèse est que la graisse périrénale est associée à la formation de lithiase urique idiopathique et que cette association dépend d'un défaut d'ammoniurie.

MATÉRIEL ET MÉTHODES : Les sujets inclus étaient des adultes de sexe masculin, avec un indice de masse corporelle supérieur ou égal à $25 \mathrm{~kg} / \mathrm{m}^{2}$ et un antécédent de lithiase urique (n = 10) ou calcique $(n=10)$ définie par spectrophotométrie infra-rouge. La quantité de graisse dans les différents compartiments abdominaux était estimée sur un scanner par la mesure des épaisseurs de graisse périrénale et des surfaces de graisse sous-cutanée et intraabdominale. Un régime standardisé ambulatoire était suivi pendant quatre jours avant de mesurer le pH urinaire, l'ammoniurie et l'excrétion nette d'acide. L'analyse statistique était réalisée par des tests de Wilcoxon.

RÉSULTATS: L'épaisseur de la graisse périrénale gauche avait tendance à être plus importante chez les sujets présentant des lithiases uriques (28 \pm 12 vs $18 \pm 8 \mathrm{~mm} ; p=0,05)$, tout comme la surface de graisse intra-abdominale qui était plus importante dans ce groupe $\left(277 \pm 88\right.$ vs $\left.185 \pm 77 \mathrm{~cm}^{2} ; p=0,01\right)$. Le $\mathrm{pH}$ urinaire était plus bas dans le groupe lithiase urique $(p=0,01)$ ainsi que le rapport ammoniurie sur excrétion urinaire nette d'acide qui avait tendance à être inférieur chez les patients présentant des lithiases uriques $(p=0,07)$.

CONCLUSION : L'acidité urinaire, la quantité de graisse périrénale et de graisse intraabdominale sont plus marquées chez les sujets présentant des lithiases uriques idiopathiques que chez ceux présentant des lithiases calciques idiopathiques. Des études complémentaires sont nécessaires pour identifier les mécanismes reliant la graisse périrénale et le défaut d’ammoniurie chez les patients lithiasiques uriques. 
LISTE DES ABRÉVIATIONS

CPP Méditerranée : Comité de Protection des Personnes Méditerranée

DFG : Débit de Filtration Glomérulaire

IMC : Indice de Masse Corporelle

IRM : Imagerie par Résonnance Magnétique

NAE : Excrétion Nette d'Acide

$\mathrm{NH}_{4}^{+}$: Ion ammonium

NHE3 : Échangeur sodium-proton $\left(\mathrm{Na}^{+} / \mathrm{H}^{+}\right)$de type 3 


\section{INTRODUCTION}

Les lithiases urinaires affectent 5 à $10 \%$ de la population dans les pays industrialisés (1). Elles sont associées à un risque cardiovasculaire plus élevé que celui de la population générale (2). Les lithiases calciques sont les plus fréquentes et les lithiases uriques représentent 10 à 20\% des patients lithiasiques. Parmi elles, les formes idiopathiques prédominent (1). Le gold standard pour identifier le type de lithiase est l'analyse du calcul par spectrophotométrie infra-rouge.

Le syndrome métabolique est défini par la présence de trois critères parmi les cinq suivants : augmentation du périmètre abdominal (dont la valeur dépend de l'ethnie et du sexe), triglycérides supérieurs ou égaux à 1,5 g/L (ou traitement spécifique), HDLcholestérol inférieur à $0,4 \mathrm{~g} / \mathrm{L}$ chez l'homme ou $0,5 \mathrm{~g} / \mathrm{L}$ chez la femme (ou traitement spécifique), tension artérielle systolique supérieure ou égale à 130 mmHg et/ou tension artérielle diastolique supérieure ou égale à $85 \mathrm{mmHg}$ (ou traitement spécifique) et augmentation de la glycémie à jeun au dessus de 1 g/L (ou traitement spécifique) (3).

Les lithiases rénales idiopathiques et le syndrome métabolique possèdent un lien épidémiologique. En effet, l'Indice de Masse Corporelle (IMC) et le diabète de type 2 sont indépendamment associés à la prévalence et l'incidence des lithiases urinaires $(4,5)$. De même, une augmentation de la fréquence des lithiases urinaires idiopathiques a été rapportée chez des sujets hypertendus (6). 
Les mécanismes responsables de la formation des lithiases uriques idiopathiques sont encore mal connus. Cependant, il existe un lien privilégié entre celles-ci et le syndrome métabolique puisque les patients lithiasiques et diabétiques de type 2 ont une plus forte prévalence de lithiases uriques que les patients lithiasiques non diabétiques $(4,7)$. Un pH urinaire bas, constamment associé aux lithiases uriques idiopathiques, est aussi retrouvé chez les sujets présentant une insulino-résistance ou diabétiques de type $2(8,9)$. De plus, l'étude de 4883 sujets lithiasiques révèle que le $\mathrm{pH}$ urinaire des $24 \mathrm{~h}$ diminue avec l'augmentation de l'IMC (10).

Les mécanismes physiopathologiques liant le syndrome métabolique, la lithiase urique idiopathique et le $\mathrm{pH}$ urinaire plus acide sont imparfaitement compris. La distribution et la composition de la graisse semblent jouer un rôle majeur. En effet, l'obésité centrale est significativement corrélée à la formation de lithiases uriques et à un $\mathrm{pH}$ urinaire acide secondaire à un défaut d'ammoniurie : il existe une corrélation négative entre le rapport graisse tronculaire/graisse des membres inférieurs, mesurée par absorptiométrie biphotonique, et l'ammoniurie évaluée par le rapport $\mathrm{NH}_{4}+/ \mathrm{NAE}$ (ammonium/excrétion acide nette) (11). L'obésité centrale, définie par des critères anthropométriques, peut aussi être mesurée de façon plus précise sur un scanner par la surface de graisse intra-abdominale (12). L'accumulation de graisse viscérale est associée à des dépôts de graisse ectopique dans de nombreux organes dont le rein (13). La stéatose rénale est définie histologiquement par une accumulation d'acides gras dans les cellules épithéliales rénales corticales. Sa quantification radiologique, par Imagerie par Résonance Magnétique (IRM) est encore à l'état de recherche. La stéatose rénale est responsable dans des modèles expérimentaux du même défaut d'ammoniurie observé chez les sujets lithiasiques uriques. La graisse périrénale pourrait être un marqueur de 
stéatose rénale $(13,14)$. La mesure de la graisse périrénale par scanner est standardisée et réalisable en pratique courante contrairement à celle de la stéatose rénale (15).

Notre hypothèse est que l'abondance de la graisse périrénale est associée à la formation de lithiases uriques idiopathiques chez l'homme et que cette association dépend d'un défaut d'ammoniurie. Pour vérifier cette hypothèse, nous avons comparé la quantité de graisse périrénale chez des sujets en surpoids ou obèses présentant des lithiases uriques idiopathiques avec la quantité de graisse périrénale de témoins présentant des lithiases calciques. Nous avons ensuite recherché un défaut d'ammoniurie chez les sujets présentant des lithiases uriques. 


\section{MATÉRIEL ET MÉTHODES}

\section{Population}

Cette étude a été conduite au Centre Hospitalier Universitaire de Nice avec l'approbation du Comité de Protection des Personnes (CPP Méditerranée). Chaque patient a signé un consentement éclairé. Les critères d'inclusion étaient le sexe masculin, l'âge supérieur à 18 ans, un surpoids ou une obésité définis par un IMC supérieur ou égal à $25 \mathrm{~kg} / \mathrm{m}^{2}$ et un antécédent de lithiase urique ou calcique. La spectrophotométrie infrarouge était utilisée pour définir les lithiases uriques (plus de 90\% d'acide urique anhydre et/ou monohydraté et/ou dihydraté) et calciques (plus de 90\% d'oxalate de calcium monohydraté et/ou dihydraté et/ou carbapatite et/ou brushite). Les sujets présentant une lithiase coralliforme, congénitale, infectieuse, de struvite ou une anomalie de la structure rénale (tumeur, kyste, amincissement cortical), une lithiase calcique ou urique secondaire, un antécédent d'hyperparathyroïdie primaire ou une hyperthyroïdie n'étaient pas inclus. Les sujets incapables de respecter le régime diététique imposé par le protocole après deux essais étaient exclus de l'analyse des critères de jugement secondaires portant sur l'excrétion rénale d'ammonium.

Les sujets étaient sélectionnés parmi les patients ayant bénéficié d’une analyse de spectrophotométrie infra-rouge de leur lithiase à Monaco et suivis dans les services d'Urologie du Centre Hospitaliser Universitaire de Nice ou du Centre Hospitalier Princesse Grace de Monaco. L'étude était proposée à tous les sujets éligibles. Ils étaient ensuite reçus en consultation de Néphrologie entre le 1er octobre 2015 et le 24 juin 2016 
pour donner leur accord écrit en signant un formulaire d'information et de nonopposition.

Recueil de données et mesures

Un scanner abdomino-pelvien était réalisé si celui-ci n'avait pas été fait dans les deux ans précédant l'inclusion ou si l'IMC avait varié de plus de 5\% depuis sa réalisation. La graisse périrénale était estimée à droite et à gauche par la mesure de son épaisseur au niveau de la veine rénale homolatérale (longueur entre la paroi postérieure du rein et la paroi antérieure de la cavité péritonéale exprimée en mm). La graisse sous-cutanée et la graisse intra-abdominale globale étaient estimées par leur surface (exprimée en $\mathrm{cm}^{2}$ ) sur la coupe de scanner passant entre la troisième et la quatrième vertèbre lombaire.

Les sujets devaient suivre un régime comprenant $400 \mathrm{mg}$ de calcium, $800 \mathrm{mg}$ de phosphore, $100 \mathrm{mmol}$ de sodium et $40 \mathrm{mmol}$ de potassium par jour et au moins 2 litres d'eau minérale de la marque Cristaline ${ }^{\circledR}$ par jour. Les apports caloriques journaliers étaient de 2700 Kcal pour les sujets de moins de 40 ans et de 2500 Kcal pour les sujets de plus de 40 ans. La consommation protéique journalière devait être de $1 \mathrm{~g} / \mathrm{kg} / \mathrm{jour}$. Aucun exercice physique n'était autorisé. L'observance au régime était évaluée après quatre jours, par une analyse des urines de $24 \mathrm{~h}$. Le respect du recueil des $24 \mathrm{~h}$ était évalué par l'excrétion urinaire de créatinine. Pour permettre d'analyser le suivi du régime, la natriurèse, la phosphaturie inorganique et l'excrétion d'urée étaient normalisées par 0,17 mmol de créatinine urinaire par kilogramme de poids corporel et par jour quel que soit l'âge. La natriurèse exprimée en mmol/24h permettait d'apprécier les apports sodés, la phosphaturie exprimée en $\mathrm{mg} / 24 \mathrm{~h}$ permettait d'apprécier les 
apports en phosphates inorganiques; enfin l'urée urinaire exprimée en mmol/24h et divisée par 5,5 fois le poids du patient permettait d'apprécier les apports protéiques alimentaires en $\mathrm{g} / \mathrm{kg} /$ jour. Si les résultats dépassaient de $10 \%$ les valeurs attendues, le sujet avait un second essai avant d'être exclu de l'analyse des critères de jugement secondaires portant sur l'excrétion rénale d'ammonium.

Après quatre à sept jours de régime imposé, les urines des $24 \mathrm{~h}$ précédentes étaient collectées (dans un récipient sous huile) et un bilan biologique a jeun était réalisé. Le pH et la $\mathrm{PCO}_{2}$ urinaires étaient mesurés à l'aide d'électrodes spécifiques et la bicarbonaturie était calculée selon l'équation d’Henderson-Hasselbach. L'appareil utilisé était un ABL 800 (Radiometer, Copenhague, Danemark). L’ammoniurie était mesurée par test UV au glutamate déshydrogénase sur l'automate Cobas 8000 (Roche Diagnostics, Mannheim, Allemagne). L'acidité titrable était mesurée manuellement par titrimétrie. La sécrétion nette d'acide (NAE) était calculée en additionnant l'ammoniurie et l'acidité titrable et en soustrayant la bicarbonaturie. Les rapports $\mathrm{NH}_{4}^{+} /$créatinine urinaire et $\mathrm{NH}_{4}{ }^{+} / \mathrm{NAE}$ étaient utilisés pour apprécier respectivement l'ammoniurie et la part de l'ammoniurie dans l'élimination des acides fixes.

Critères de Jugement

Le critère de jugement principal était l'épaisseur de la graisse périrénale gauche associée aux lithiases uriques par rapport à celle associée aux lithiases calciques. Les critères de jugement secondaires étaient l'épaisseur de la graisse périrénale droite, la surface de la graisse sous-cutanée et la surface de la graisse intra-abdominale globale 
dans les deux groupes ainsi que le $\mathrm{pH}$ et l'excrétion rénale d'ammonium des $24 \mathrm{~h}$ rapportée à l'excrétion nette d'acide ou à la créatinine après le régime standardisé.

\section{Analyses Statistiques}

Le nombre de sujets nécessaires pour obtenir une puissance de $80 \%$ et un risque alpha de 5\% était de 10 dans chaque groupe. Les comparaisons quantitatives ont été réalisées à l'aide d'un test de Wilcoxon. Les analyses statistiques ont été réalisées avec le logiciel R. Les résultats sont exprimés en moyenne \pm écart-type. La significativité statistique est définie par $p<0,05$. 


\section{RÉSULTATS}

Le tableau 1 reprend les caractéristiques de la population étudiée. Dix sujets ont été inclus dans le groupe lithiase calcique et dix dans le groupe lithiase urique. Les IMC moyens étaient respectivement de $30 \pm 2,9 \mathrm{~kg} / \mathrm{m}^{2}$ et de $28,4 \pm 3,1 \mathrm{~kg} / \mathrm{m}^{2}$. L'âge moyen était de $59 \pm 10$ ans dans le groupe lithiase calcique et $64 \pm 9$ ans dans le groupe lithiase urique. La fonction rénale était comparable dans les deux groupes. Un sujet du groupe lithiase calcique présentait un diabète de type 2 contre six dans le groupe lithiase urique. L'ancienneté d'évolution du diabète était de 11 et $6 \pm 3$ ans respectivement dans les groupes lithiases calciques et uriques.

\begin{tabular}{|c|c|c|}
\hline & Lithiase Calcique & Lithiase Urique \\
\hline Nombre & 10 & 10 \\
\hline IMC $\left(\mathrm{kg} / \mathrm{m}^{2}\right)$ & $30 \pm 2,9$ & $28,4 \pm 3,1$ \\
\hline Âge (ans) & $59 \pm 10$ & $64 \pm 9$ \\
\hline Créatinine ( $\mu \mathrm{mol} / \mathrm{L})$ & $82 \pm 9$ & $91 \pm 18$ \\
\hline DFG (mL/min $\left./ 1,73 \mathrm{~m}^{2}\right)$ & $88 \pm 14$ & $76 \pm 18$ \\
\hline Diabète de type 2 (nombre) & 1 & 6 \\
\hline Ancienneté d'évolution du diabète (ans) & 11 & $6 \pm 3$ \\
\hline
\end{tabular}

Tableau 1. Caractéristiques générales de la population.

Les résultats sont exprimés selon la forme suivante : moyenne \pm écart-type.

IMC : Indice de Masse Corporelle. DFG : Débit de Filtration Glomérulaire.

Le tableau 2 regroupe les mesures de graisse périrénale, sous-cutanée et intraabdominale globale dans les deux groupes. L'épaisseur de la graisse périrénale gauche était de 18,1 \pm 7,8 $\mathrm{mm}$ dans le groupe lithiase calcique et $27,9 \pm 11,7 \mathrm{~mm}$ dans le groupe lithiase urique. L'analyse statistique montre une tendance à une plus importante épaisseur de la graisse périrénale gauche chez les patients présentant des lithiases uriques avec un $p$ égal à 0,05. Ces données sont représentées sur la figure 1. 
L'épaisseur de la graisse périrénale droite n'était pas significativement différente dans les deux groupes avec des épaisseurs respectives de 17,3 $\pm 9,2 \mathrm{~mm}$ et $25,7 \pm 11,2 \mathrm{~mm}$ dans les groupes lithiase calcique et urique. De même, la surface de graisse sous-cutanée n'était pas différente dans les deux groupes. En revanche, la surface de la graisse intraabdominale globale était plus importante chez les sujets lithiasiques uriques que chez les sujets présentant des lithiases calciques $(p=0,01)$.

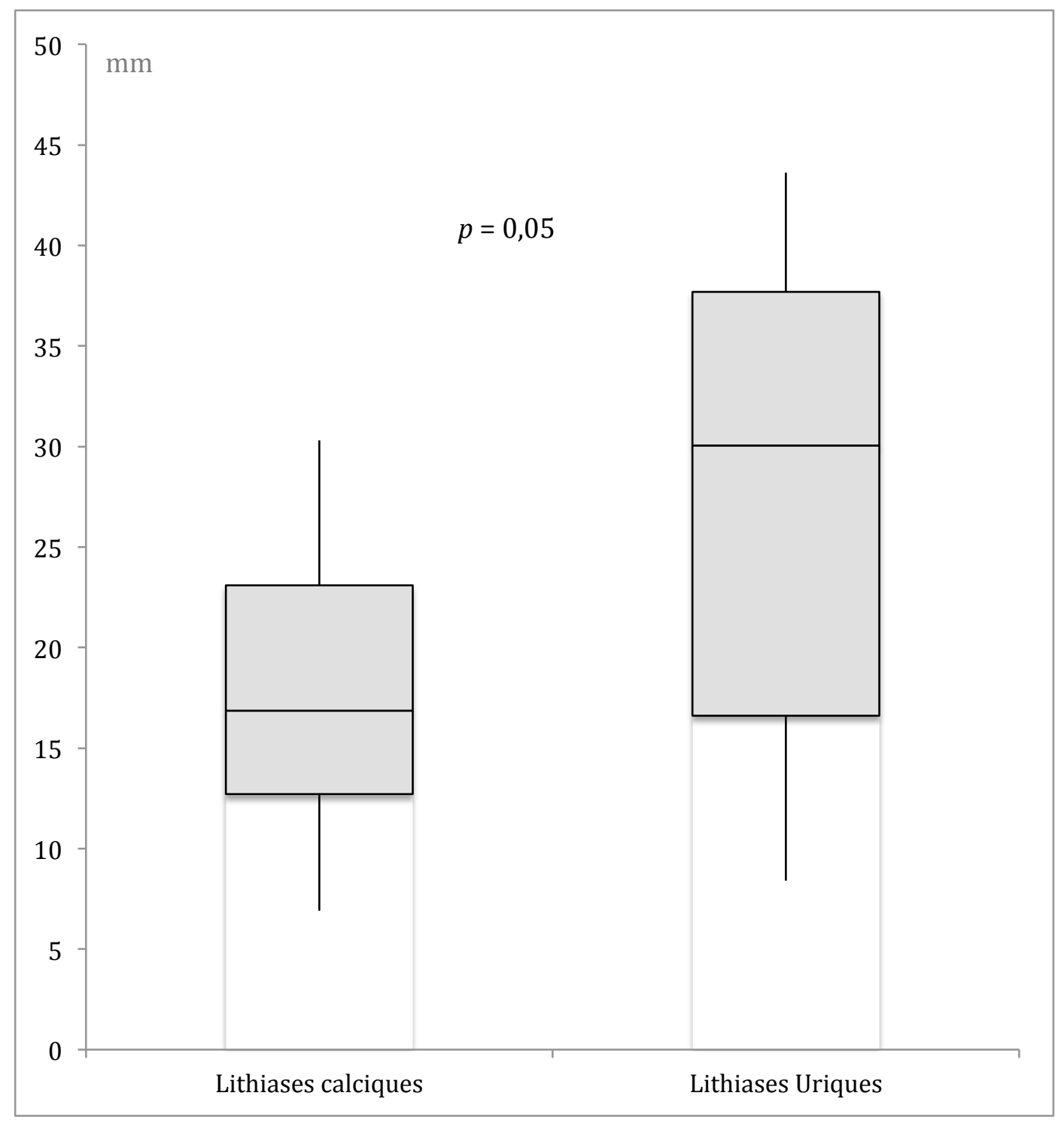

Figure 1. Épaisseur de la graisse périrénale gauche dans les deux groupes. 


\begin{tabular}{lccc}
\hline & Lithiase Calcique & Lithiase Urique & $\boldsymbol{p}$ \\
\hline Nombre & 10 & 10 & \\
& & & \\
Épaisseur en mm & & & \\
$\quad$ Graisse Périrénale Gauche & $18,1 \pm 7,8$ & $27,9 \pm 11,7$ & 0,05 \\
$\quad$ Graisse Périrénale Droite & $17,3 \pm 9,2$ & $25,7 \pm 11,2$ & 0,15 \\
& & & \\
Surface en cm${ }^{2}$ & & $182,8 \pm 65,7$ & 0,28 \\
$\quad$ Graisse Sous-Cutanée & $247,1 \pm 115,4$ & $276,6 \pm 88,2$ & 0,01 \\
$\quad$ Graisse Intra-Abdominale Globale & $185,1 \pm 77,2$ & & \\
\hline
\end{tabular}

Tableau 2. Mesures de graisse périrénale, sous-cutanée et intra-abdominale globale Les résultats sont exprimés selon la forme suivante : moyenne \pm écart-type

Parmi les 20 sujets inclus, seuls 13 ont suivi le régime permettant d'interpréter les paramètres urinaires. Cinq n'ont pas accepté de réaliser le régime, le plus souvent par manque de disponibilité et deux sujets n'ont pas réussi à suivre le régime malgré les deux essais et ont été exclus de l'analyse des paramètres urinaires. Treize sujets ont suivi l'intégralité du protocole, 7 dans le groupe lithiase calcique et 6 dans le groupe lithiase urique.

Après quatre jours de régime contrôlé, il n’y avait pas de différence significative entre les deux groupes pour l'excrétion urinaire d'urée reflétant les apports alimentaires en protéines, l'excrétion de phosphates inorganiques intervenant dans l'acidité titrable, la natriurèse correspondant aux apports sodées et la diurèse. Ces données sont reprises dans le tableau 3. 


\begin{tabular}{lccc}
\hline & Lithiase Calcique & Lithiase Urique & $\boldsymbol{p}$ \\
\hline Nombre & 7 & 6 & \\
Apport Protéique (g/kg/jour) & $0,9 \pm 0,2$ & $0,8 \pm 0,3$ & 0,17 \\
Phosphaturie (mg/jour) & $685 \pm 226,6$ & $741,3 \pm 171,8$ & 0,95 \\
Natriurèse (mmol/jour) & $102 \pm 39$ & $119 \pm 26$ & 0,29 \\
Diurèse (litres/jour) & $2,7 \pm 1,2$ & $2,1 \pm 0,7$ & 0,28
\end{tabular}

Tableau 3. Respect du régime alimentaire.

Les résultats sont exprimés selon la forme suivante : moyenne \pm écart-type

Les résultats des explorations urinaires sont exposés dans le tableau 4. Le pH urinaire des $24 \mathrm{~h}$ des sujets présentant des lithiases uriques était inférieur à celui des sujets avec des lithiases calciques $(5,30 \pm 0,36$ dans le groupe lithiase urique et 6,04 \pm 0,44 dans le groupe lithiase calcique) $(p=0,01)$. L'excrétion urinaire d'ammonium $\left(\mathrm{NH}_{4}{ }^{+}\right)$par rapport à l'excrétion nette d'acide (NAE) avait une tendance statistique à être plus basse dans le groupe des sujets présentant des lithiases uriques $(p=0,07)$ : l'excrétion urinaire d'ammonium représentait 47,6 \pm 12,2\% de l'excrétion nette d'acide dans le groupe lithiase urique contre $66,9 \pm 16,7 \%$ dans le groupe lithiase calcique. L'excrétion urinaire d'ammonium standardisée à la créatinine urinaire des $24 \mathrm{~h}$ était de $1,96 \pm 0,72 \mathrm{mmol} / \mathrm{mmol}$ dans le groupe lithiase urique et de $3,06 \pm 1,35 \mathrm{mmol} / \mathrm{mmol}$ dans le groupe lithiase calcique. Il n'y avait pas de différence significative pour ce critère.

\begin{tabular}{lccc}
\hline & Lithiase Calcique & Lithiase Urique & $\boldsymbol{p}$ \\
\hline Nombre & 7 & 6 & \\
pH urinaire des 24h & $6,04 \pm 0,44$ & $5,30 \pm 0,36$ & 0,01 \\
$\mathbf{N H}_{4}{ }^{+}$NAE urinaire des $\mathbf{2 4 h}$ & $0,669 \pm 0,167$ & $0,476 \pm 0,122$ & 0,07 \\
$\mathbf{N H}_{4}+$ créatinine urinaire des 24h & $3,06 \pm 1,35$ & $1,96 \pm 0,72$ & 0,18 \\
\end{tabular}

Tableau 4. Explorations urinaires.

Les résultats sont exprimés selon la forme suivante : moyenne \pm écart-type. 


\section{DISCUSSION}

Le résultat original de notre étude est que pour un même IMC, les sujets lithiasiques uriques présentent plus de graisse périrénale que les sujets lithiasiques calciques $(p=0,05)$. Notre second résultat est que la quantité de graisse intraabdominale est plus importante chez les sujets présentant des lithiases uriques par rapport à ceux présentant des lithiases calciques. Ces deux résultats sont cohérents puisque l'abondance de la graisse périrénale est corrélée à celle de la graisse intraabdominale $(16,17)$. L'association entre graisse intra-abdominale et lithiase urique est aussi retrouvée par Kim et al, chez 36 patients coréens (18).

Aucune différence significative de surface de graisse sous cutanée n’est retrouvée entre les deux groupes dans notre étude. Ce résultat est conforté par le fait qu'il n’y a pas de corrélation entre le volume de graisse périrénale et la surface de graisse souscutanée dans la littérature chez l'individu de sexe masculin $(14,16)$.

Le $\mathrm{pH}$ urinaire des sujets lithiasiques uriques est significativement plus bas que celui des sujets lithiasiques calciques, toutes caractéristiques égales par ailleurs (tableau 4). En contrôlant le régime alimentaire pendant au moins quatre jours dans des conditions de vie normales, on constate que le défaut d'ammoniurie explique l'acidité urinaire. En effet, le rapport $\mathrm{NH}_{4}+\mathrm{NAE}$ a tendance à être plus bas chez les sujets lithiasiques uriques que chez ceux présentant des lithiases calciques et cela n'est pas dû à une différence d'acidité titrable (sulfates et phosphates alimentaires) ni à une différence d'ammoniogenèse puisque la charge acide alimentaire est comparable entre 
les deux groupes (tableaux 3 et 4). La production d'urine acide secondaire à un défaut d'ammoniurie chez les sujets présentant des lithiases uriques est décrite dans la littérature $(7,19)$. De manière remarquable, l'analyse des paramètres urinaires de sujets non lithiasiques diabétiques de type 2 (9 sujets comparés à 16 sujets témoins) ou présentant un syndrome métabolique (148 sujets) retrouve une acidité urinaire secondaire à un défaut d'ammoniurie comme chez les sujets lithiasiques uriques $(9,20)$. Il est important de noter que les patients de notre étude cumulent ces différents traits puisqu'ils sont à la fois en surpoids ou obèses et lithiasiques. Nous observons d'ailleurs davantage de sujets diabétiques dans notre étude dans le groupe lithiase urique (tableau 1). Sur la base d'analyses morpho-constitutionnelles de plus de 2000 calculs urinaires, il a été montré que la prévalence du diabète de type 2 est plus élevée chez les sujets présentant des lithiases uriques (4).

Le question du mécanisme liant l'excès de graisse viscérale au défaut d'ammoniurie se pose. Le surpoids et l'obésité sont responsables d'une inflammation chronique de bas grade par modification du profil histologique et sécrétoire de la graisse. Des adipocytokines sont sécrétées par ce tissu adipeux inflammatoire comportant des adipocytes hypertrophiés et des macrophages de type M1 (21). La graisse périrénale est anatomiquement mieux placée que la graisse intra-abdominale globale pour produire des effets néfastes sur le parenchyme rénal. De plus, il existe des associations déjà connues entre la graisse périrénale et les différentes fonctions rénales (équilibre hydro-sodé, albuminurie d'effort et maladie rénale chronique). Ces associations à la graisse périrénale sont indépendantes du volume de graisse intraabdominale $(16,17,22)$. Deux mécanismes de toxicité de la graisse périrénale peuvent être évoqués : une toxicité paracrine de par sa localisation anatomique périrénale, ou 
une toxicité endocrine à travers le plexus artériel sub-péritonéal de Turner qui est responsable de 6\% de la vascularisation rénale corticale (23). Parmi les différentes adipocytokines, les acides gras intra-rénaux semblent être impliqués dans la diminution de l'ammoniurie. En effet, la stéatose rénale, qui est définie par une accumulation d'acides gras dans les cellules épithéliales rénales corticales provoque un défaut d'ammoniurie. Bobulescu et al, ont mis en évidence une diminution du pH et de l'ammoniurie chez des rats obèses et diabétiques (Zucker) présentant une stéatose rénale. Ces anomalies sont secondaires à une diminution de l'expression et de l'activité des échangeurs membranaires sodium/proton de type 3 (NHE3) de la bordure en brosse tubulaire rénale (24). Ces anomalies sont réversibles chez le rat. En effet, après un traitement de quatre semaines par des glitazones (Thiazolidinedione), on note une diminution de la stéatose rénale et on observe une normalisation des paramètres urinaires (25). La supplémentation en vitamine D active (Doxercalciferol ou $1 \alpha$ hydroxyvitamine $\mathrm{D}_{2}$ ) prévient l'apparition de la stéatose rénale chez la souris exposée à une alimentation hypercalorique. La stimulation des facteurs de transcription PPAR $\gamma$ et SREBP-1, respectivement par les glitazones et la vitamine $\mathrm{D}$ active, diminue expérimentalement la stéatose rénale $(25,26)$. Un argument supplémentaire en faveur d'une toxicité directe des acides gras est que l'ajout d'acides gras à longues chaînes sur des cellules rénales d'opossum ex-vivo diminue l'activité des échangeurs membranaires NHE3 stimulés par l'insuline (25).

La graisse périrénale pourrait être un marqueur de stéatose rénale. En effet, chez l’homme de sexe masculin, la graisse périrénale est corrélée positivement au périmètre abdominal tandis que l'IMC est corrélé positivement à la stéatose rénale mesurée par le dosage des triglycérides corticaux sur des pièces de néphrectomie $(13,14)$. Cette 
corrélation entre stéatose rénale et graisse périrénale existe chez des cochons que l'on expose à un régime hypercalorique pendant dix semaines pour reproduire les effets d'un syndrome métabolique. Une augmentation conjointe de la graisse périrénale et de la stéatose rénale est alors observée et mesurée sur des prélèvements tissulaires (27). Dans un futur proche, nous analyserons la corrélation entre la stéatose rénale histologique et le pourcentage de graisse dans le cortex rénal mesuré par IRM. Nous réaliserons la mesure de la stéatose rénale par dosage des triglycérides corticaux grâce à un marquage à l'oil-red-0 sur des pièces de néphrectomie prélevées chez des patients atteints de cancer du rein. Cela nous permettra de rechercher ensuite une association directe entre défaut d'ammoniurie et stéatose rénale chez les patients de notre étude. De plus, nous rechercherons si cette association est spécifique de la lithiase urique. Si cette association entre stéatose rénale et défaut d'ammoniurie était confirmée chez les patients atteints de lithiase urique idiopathique, il serait envisageable de tester l'effet de la supplémentation par vitamine D active sur la stéatose rénale qui serait considérée comme une cible thérapeutique dans la prise en charge de la lithiase urique idiopathique chez l'homme.

La sélection des patients sur l'analyse des lithiases par spectrophotométrie infrarouge est l'une des forces de notre étude. En effet, il s'agit du seul moyen d'identifier précisément le type de lithiase, les méthodes d'analyses urinaires et sanguines étant imprécises. De même, la mesure des compartiments graisseux par scanner à l'aide d'une méthode manuelle permet une quantification précise de la graisse par rapport à la méthode automatisée par seuillage en raison de l’hétérogénéité de densité interindividuelle de la graisse au scanner (15). De plus, le choix de l'épaisseur de la graisse périrénale pour l'estimation de la quantité de graisse périrénale est le 
paramètre le mieux corrélé au volume de cette graisse et présentant la plus faible variabilité inter-opérateur (14). Une limite de notre étude est la difficulté du suivi du régime alimentaire standardisé en ambulatoire. En effet, nos résultats concernant le rapport $\mathrm{NH}_{4}{ }^{+} / \mathrm{NAE}$ révèlent une tendance mais n'atteignent pas la significativité (tableau 4) contrairement à ceux de Sakhaee et al, qui délivrent un régime standardisé en milieu hospitalier dans leur étude (19). De plus, nous ne disposons que de données chez des sujets de sexe masculin qui ne sont pas extrapolables chez la femme du fait d'importantes différences de répartition des graisses entre les deux sexes (28). Cependant les femmes, comme les hommes, présentent l'association lithiase urique et syndrome métabolique (4). Enfin, nous rapportons des associations entre graisse périrénale et défaut d'ammoniurie chez les sujets lithiasiques uriques mais pour affirmer que cette association est indépendante de la graisse intra-abdominale, des analyses complémentaires sont nécessaires et sont en cours. Nous réaliserons une régression linéaire, avec l'épaisseur de la graisse périrénale comme variable à expliquer, le groupe de lithiase (urique ou calcique) comme variable explicative d'intérêt et la surface de graisse intra-abdominale comme variable d'ajustement. 
CONCLUSION

La quantité de graisse périrénale et l'acidité urinaire secondaire au défaut d'ammoniurie sont caractéristiques des sujets présentant des lithiases uriques idiopathiques. Cela suggère un lien physiopathologique entre tissu adipeux et défaut d'ammoniurie. Plus précisément, la toxicité des acides gras libres intra-rénaux sur l'ammoniurie via l'expression et l'activité des échangeurs NHE3 tubulaires proximaux font penser que la stéatose rénale pourrait favoriser la lithogenèse urique chez l'homme. La graisse périrénale pourrait être un marqueur de stéatose rénale facilement mesurable. Cette relation reste à démontrer chez l'homme avant d'envisager des études interventionnelles ciblant la stéatose rénale. 


\section{BIBLIOGRAPHIE}

1. Bartoletti R, Cai T, Mondaini N, Melone F, Travaglini F, Carini M, et al. Epidemiology and Risk Factors in Urolithiasis. Urologia Internationalis. 2007;79(1):3-7.

2. Raynal G, Achkar K, El Samad R, Kikassa J-C, Jorest R. Risque cardiovasculaire chez les lithiasiques. Progrès en Urologie. 2008 May;18(5):288-91.

3. Alberti KGMM, Eckel RH, Grundy SM, Zimmet PZ, Cleeman JI, Donato KA, et al. Harmonizing the Metabolic Syndrome: A Joint Interim Statement of the International Diabetes Federation Task Force on Epidemiology and Prevention; National Heart, Lung, and Blood Institute; American Heart Association; World Heart Federation; International Atherosclerosis Society; and International Association for the Study of Obesity. Circulation. 2009 Oct 20;120(16):1640-5.

4. Daudon M. Type 2 Diabetes Increases the Risk for Uric Acid Stones. Journal of the American Society of Nephrology. 2006 Jun 21;17(7):2026-33.

5. Curhan GC, Willett WC, Rimm EB, Speizer FE, Stampfer MJ. Body size and risk of kidney stones. Journal of the American Society of Nephrology. 1998;9(9):1645-52.

6. Ramello A, Vitale C, Marangella M. Epidemiology of nephrolithiasis. J Nephrol. 2000 Dec;13 Suppl 3:S45-50.

7. Pak CY, Sakhaee K, Peterson RD, Poindexter JR, Frawley WH. Biochemical profile of idiopathic uric acid nephrolithiasis. Kidney international. 2001;60(2):757-61.

8. Abate N, Chandalia M, Cabo-Chan AV, Moe OW, Sakhaee K. The metabolic syndrome and uric acid nephrolithiasis: novel features of renal manifestation of insulin resistance. Kidney international. 2004;65(2):386-92.

9. Maalouf NM, Cameron MA, Moe OW, Adams-Huet B, Sakhaee K. Low Urine pH: A Novel Feature of the Metabolic Syndrome. Clinical Journal of the American Society of Nephrology. 2007 Aug 16;2(5):883-8.

10. Maalouf NM, Sakhaee K, Parks JH, Coe FL, Adams-Huet B, Pak CY. Association of urinary pH with body weight in nephrolithiasis. Kidney international. 2004;65(4):14225.

11. Pigna F, Sakhaee K, Adams-Huet B, Maalouf NM. Body Fat Content and Distribution and Urinary Risk Factors for Nephrolithiasis. Clinical Journal of the American Society of Nephrology. 2014 Jan 7;9(1):159-65.

12. Fox CS, Massaro JM, Hoffmann U, Pou KM, Maurovich-Horvat P, Liu C-Y, et al. Abdominal Visceral and Subcutaneous Adipose Tissue Compartments: Association With Metabolic Risk Factors in the Framingham Heart Study. Circulation. 2007 Jul 3;116(1):39-48. 
13. Bobulescu IA, Lotan Y, Zhang J, Rosenthal TR, Rogers JT, Adams-Huet B, et al. Triglycerides in the Human Kidney Cortex: Relationship with Body Size. Norata GD, editor. PLoS ONE. 2014 Aug 29;9(8):e101285.

14. Favre G, Grangeon-Chapon C, Raffaelli C, Chalmin F, Iannelli A, Esnault V. Relevance of perirenal fat volume measures. PLOS ONE. (En révision);

15. Yoshizumi T, Nakamura T, Yamane M, Waliul Islam AHM, Menju M, Yamasaki K, et al. Abdominal fat: Standardized technique for measurement at ct 1 . Radiology. 1999;211(1):283-6.

16. Chughtai HL, Morgan TM, Rocco M, Stacey B, Brinkley TE, Ding J, et al. Renal Sinus Fat and Poor Blood Pressure Control in Middle-Aged and Elderly Individuals at Risk for Cardiovascular Events. Hypertension. 2010 Nov 1;56(5):901-6.

17. Foster MC, Hwang S-J, Porter SA, Massaro JM, Hoffmann U, Fox CS. Fatty Kidney, Hypertension, and Chronic Kidney Disease: The Framingham Heart Study. Hypertension. 2011 Nov 1;58(5):784-90.

18. Kim JH, Doo SW, Cho KS, Yang WJ, Song YS, Hwang J, et al. Which anthropometric measurements including visceral fat, subcutaneous fat, body mass index, and waist circumference could predict the urinary stone composition most? BMC Urology. 2015 Dec;15(1).

19. Sakhaee K, Adams-Huet B, Moe OW, Pak CY. Pathophysiologic basis for normouricosuric uric acid nephrolithiasis. Kidney international. 2002;62(3):971-9.

20. Maalouf NM, Cameron MA, Moe OW, Sakhaee K. Metabolic Basis for Low Urine pH in Type 2 Diabetes. Clinical Journal of the American Society of Nephrology. 2010 Jul 1;5(7):1277-81.

21. Weisberg SP, McCann D, Desai M, Rosenbaum M, Leibel RL, Ferrante AW. Obesity is associated with macrophage accumulation in adipose tissue. Journal of Clinical Investigation. 2003 Dec 15;112(12):1796-808.

22. Wagner R, Machann J, Lehmann R, Rittig K, Schick F, Lenhart J, et al. Exerciseinduced albuminuria is associated with perivascular renal sinus fat in individuals at increased risk of type 2 diabetes. Diabetologia. 2012 Jul;55(7):2054-8.

23. Shoja MM, Shane Tubbs R, Loukas M, Shokouhi G, Ghabili K, Agutter PS. The subperitoneal arterial plexus of Sir William Turner. Annals of Anatomy - Anatomischer Anzeiger. 2010 Aug;192(4):194-8.

24. Bobulescu IA, Dubree M, Zhang J, McLeroy P, Moe OW. Effect of renal lipid accumulation on proximal tubule $\mathrm{Na}+\mathrm{H}+$ exchange and ammonium secretion. AJP: Renal Physiology. 2008 Apr 2;294(6):F1315-22. 
25. Bobulescu IA, Dubree M, Zhang J, McLeroy P, Moe OW. Reduction of renal triglyceride accumulation: effects on proximal tubule $\mathrm{Na}+\mathrm{H}+$ exchange and urinary acidification. AJP: Renal Physiology. 2009 Nov 1;297(5):F1419-26.

26. Wang XX, Jiang T, Shen Y, Santamaria H, Solis N, Arbeeny C, et al. Vitamin D receptor agonist doxercalciferol modulates dietary fat-induced renal disease and renal lipid metabolism. AJP: Renal Physiology. 2011 Mar 1;300(3):F801-10.

27. Li Z, Woollard JR, Wang S, Korsmo MJ, Ebrahimi B, Grande JP, et al. Increased glomerular filtration rate in early metabolic syndrome is associated with renal adiposity and microvascular proliferation. AJP: Renal Physiology. 2011 Nov 1;301(5):F1078-87.

28. Eisner BH, Zargooshi J, Berger AD, Cooperberg MR, Doyle SM, Sheth S, et al. Gender differences in subcutaneous and perirenal fat distribution. Surgical and Radiologic Anatomy. 2010 Nov;32(9):879-82. 


\section{SERMENT D'HIPPOCRATE}

Au moment d'être admis(e) à exercer la médecine, je promets et je jure d'être fidèle aux lois de l'honneur et de la probité.

Mon premier souci sera de rétablir, de préserver ou de promouvoir la santé dans tous ses éléments, physiques et mentaux, individuels et sociaux.

Je respecterai toutes les personnes, leur autonomie et leur volonté, sans aucune discrimination selon leur état ou leurs convictions. J'interviendrai pour les protéger si elles sont affaiblies, vulnérables ou menacées dans leur intégrité ou leur dignité. Même sous la contrainte, je ne ferai pas usage de mes connaissances contre les lois de l'humanité.

J'informerai les patients des décisions envisagées, de leurs raisons et de leurs conséquences.

Je ne tromperai jamais leur confiance et n'exploiterai pas le pouvoir hérité des circonstances pour forcer les consciences.

Je donnerai mes soins à l'indigent et à quiconque me les demandera. Je ne me laisserai pas influencer par la soif du gain ou la recherche de la gloire.

Admis(e) dans l'intimité des personnes, je tairai les secrets qui me seront confiés. Reçu(e) à l'intérieur des maisons, je respecterai les secrets des foyers et ma conduite ne servira pas à corrompre les moeurs.

Je ferai tout pour soulager les souffrances. Je ne prolongerai pas abusivement les agonies. Je ne provoquerai jamais la mort délibérément.

Je préserverai l'indépendance nécessaire à l'accomplissement de ma mission. Je n'entreprendrai rien qui dépasse mes compétences. Je les entretiendrai et les perfectionnerai pour assurer au mieux les services qui me seront demandés.

J'apporterai mon aide à mes confrères ainsi qu'à leurs familles dans l'adversité.

Que les hommes et mes confrères m'accordent leur estime si je suis fidèle à mes promesses ; que je sois déshonoré(e) et méprisé(e) si j’y manque. 